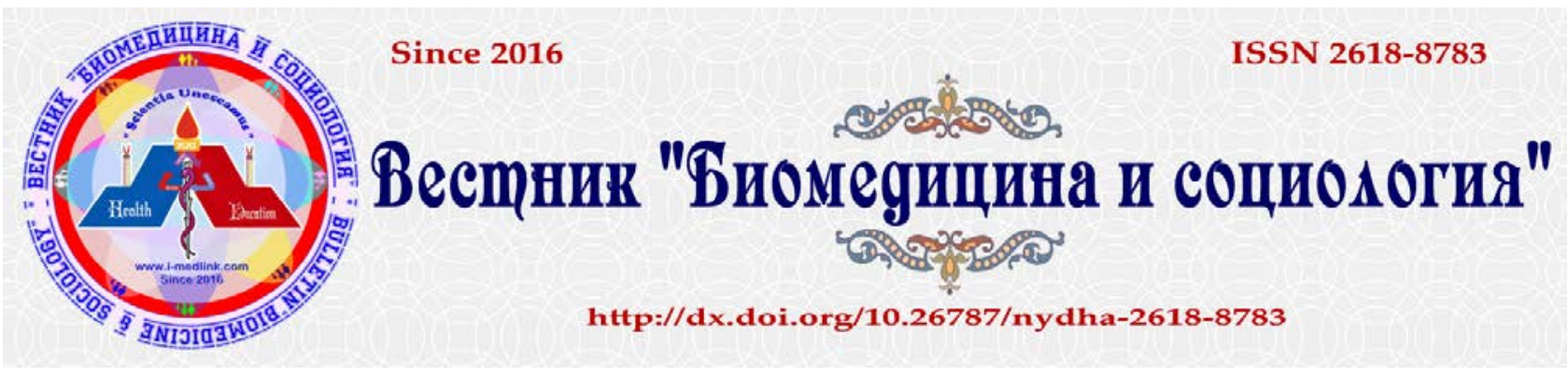

2018, том 3 [4]

http://dx.doi.org/10.26787/nydha-2618-8783-2018-3-4-17-19

\title{
ENDOCRINE ASPECTS OF MALE INFERTILITY
}

\author{
Akopova R.A., Kokoreva T.V. \\ RUDN University, Moscow, Russian Federation
}

\begin{abstract}
Annotation: endocrine disorders are widespread in men with infertility.
Objective: to assess the prognostic significance of follicle stimulating hormone (FSH) in terms of spermatogenesis in infertile men.

Materials and methods. Surveyed 150 men with impaired semen analysis. All patients were measured FSH level, the volume of the testicle.

Results. Normal indicators of FSH (12-15) IU/ml were in 96(64\%). The level of FSH, sperm motility and testicular volume show an inverse relationship $(\mathrm{p}<0,05)$.

Summary. Endocrine disorders occur in 36\% of men with infertility.
\end{abstract}

Key words: male infertility, asthenozoospermia, FSH.

The term «infertility» refers to the inability of a couple of childbearing age to conceive a child with a regular sexual life for 12 months without the use of contraceptives [1, 2, 5]. Today in the world there are about $10-15 \%$ of couples of reproductive age [4]. Only the male factor causes about $30 \%$ of infertility cases, while the combination of male and female factors is detected in $20 \%$ of cases [5]. Therefore, the overall male infertility factor will be present in $50 \%$ [3].

The pathophysiology of male infertility addresses several issues: genetic/congenital anomalies, postinfectious problems, varicocele, injuries, endocrine disorders [4]. Despite the fact that pathospermia may indicate a violation of spermatogenesis throughout the testicle, clinical cases prove that in half of the cases spermatozoa can be detected in some loci [3]. That gives hope for their successful extraction during the assisted reproductive technologies.

Therefore, the evaluation of serum levels of follicle stimulating hormone (FSH) is used by modern andrologists as a marker of spermatogenesis.

Objective: to assess the prognostic significance of FSH in terms of spermatogenesis in infertile men.

Materials and methods: 150 men with sperm disorders were examined. All patients were produced:

- measurement of height and body weight, waist circumference and body mass index calculation;

- evaluation of the proportions of the skeleton (eunuchoid proportions, reduced growth);

- inspection of androgen-dependent zones (hair);

- examination of the scrotal organs (palpation and assessment of testicular volume, detection of testicular tumors, the presence of the VAS deferens and the study of the state of the epididymis);

- assessment of hormonal status (testosterone, FSH and LH);

- karyotyping.

The statistical analysis was performed using spreadsheets «EXCEL» and «STATISTICA 6.0». The significance of differences between quantitative indicators was assessed using the Mann - Whitney test. To compare the qualitative parameters, the exact Fisher or $\chi 2$ criterion was used. Differences were considered significant at $\mathrm{p}<0,05$.

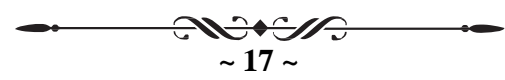

Вестник представлен в научной электронной библиотеке (НЭБ) - головном исполнителе проекта по созданию Российского индекса научного цитирования (РИНЦ) 
Results: the Average age of patients who underwent sperm collection from the testicles was 35,4 $\pm 2,7$ years (from 26 to 45 years).

Indicators of FSH level in examined patients are shown in figure 1. Normal indicators of FSH (1215) $\mathrm{IU} / \mathrm{ml}$ were in 96 (64\%). In the remaining men (36\%), we recorded a deviation in FSH due to endocrine disorders.

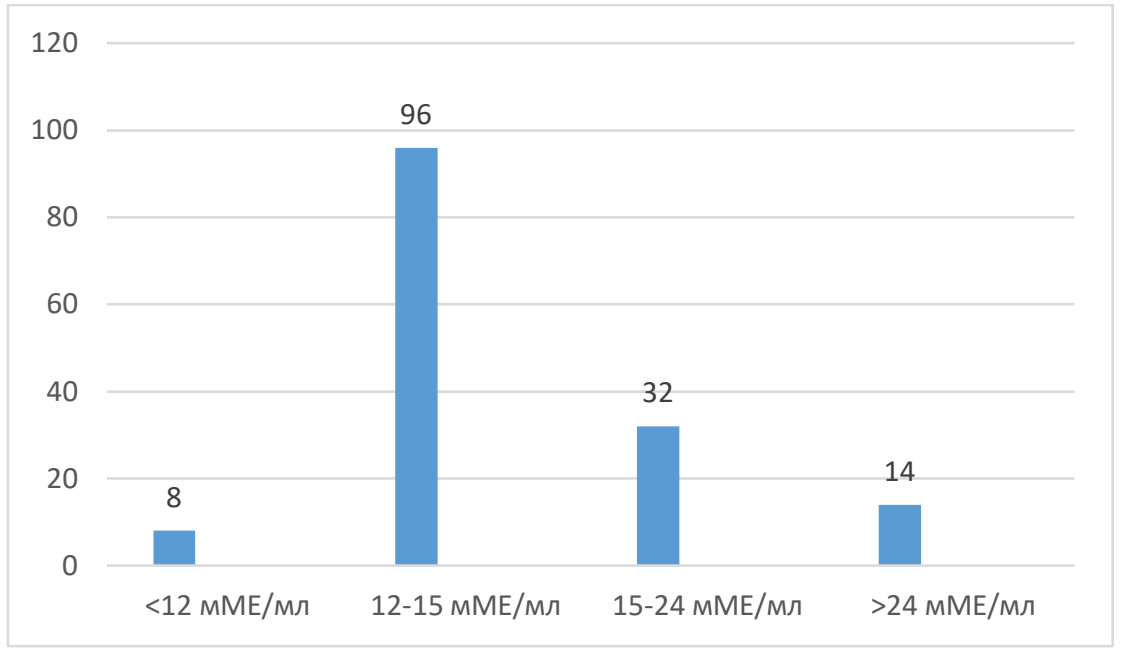

Figure 1. Indicators of FSH level in examined patients.

Levels of FSH and semen are presented in figure 2. The level of FSH and sperm motility demonstrate an inverse relationship $(\mathrm{p}<0,05)$.

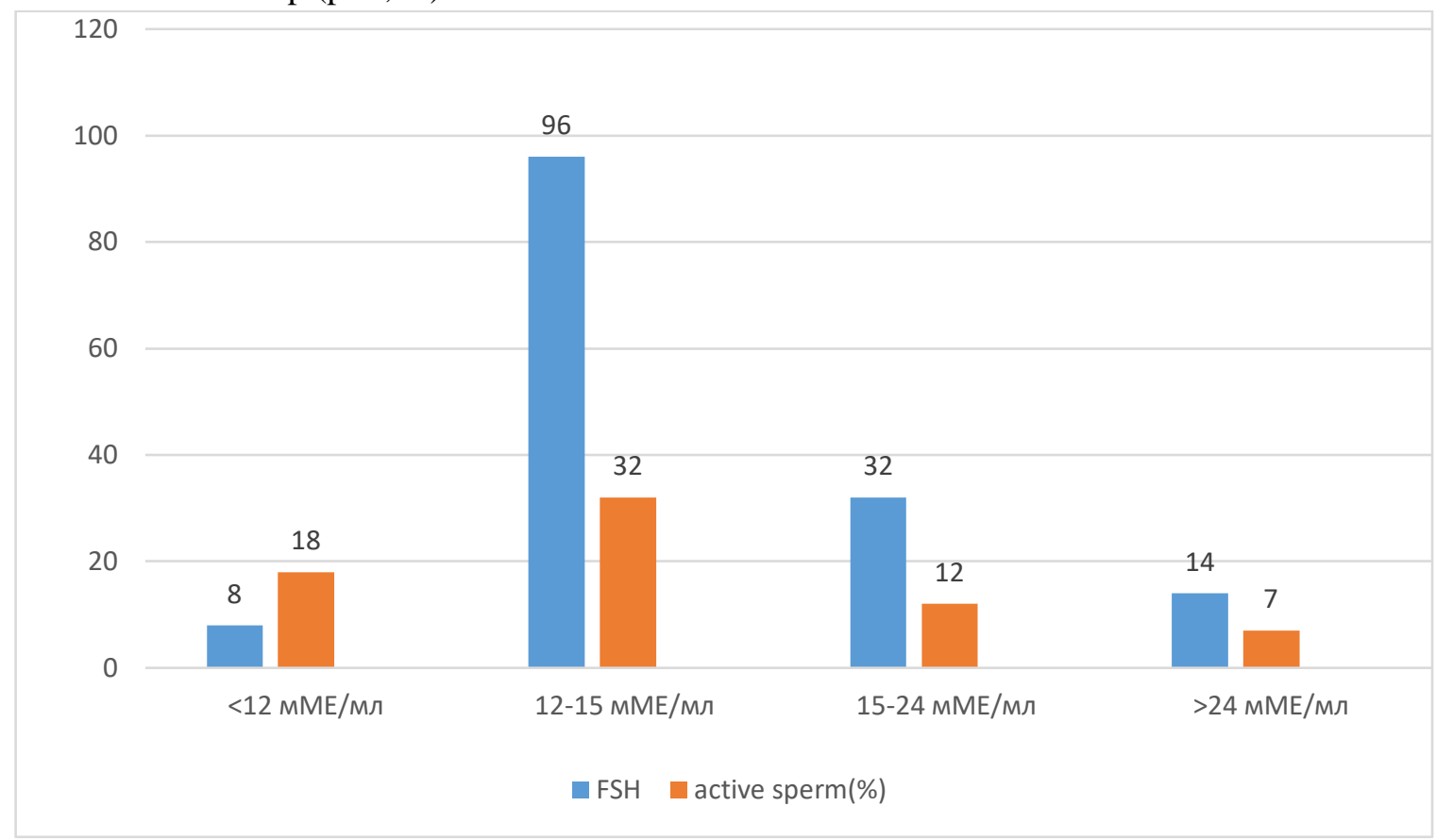

Figure 2. Levels of FSH and semen.

Indicators of FSH level and testicular volume are shown in figure 3. We found the inverse relationship between FSH level and testicular volume $(\mathrm{p}<0,05)$.

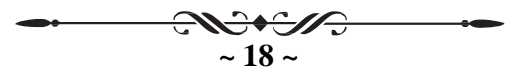


On line scientific journal - Bulletin "Biomedicine \& sociology", 2018. Vol. 3. No 4

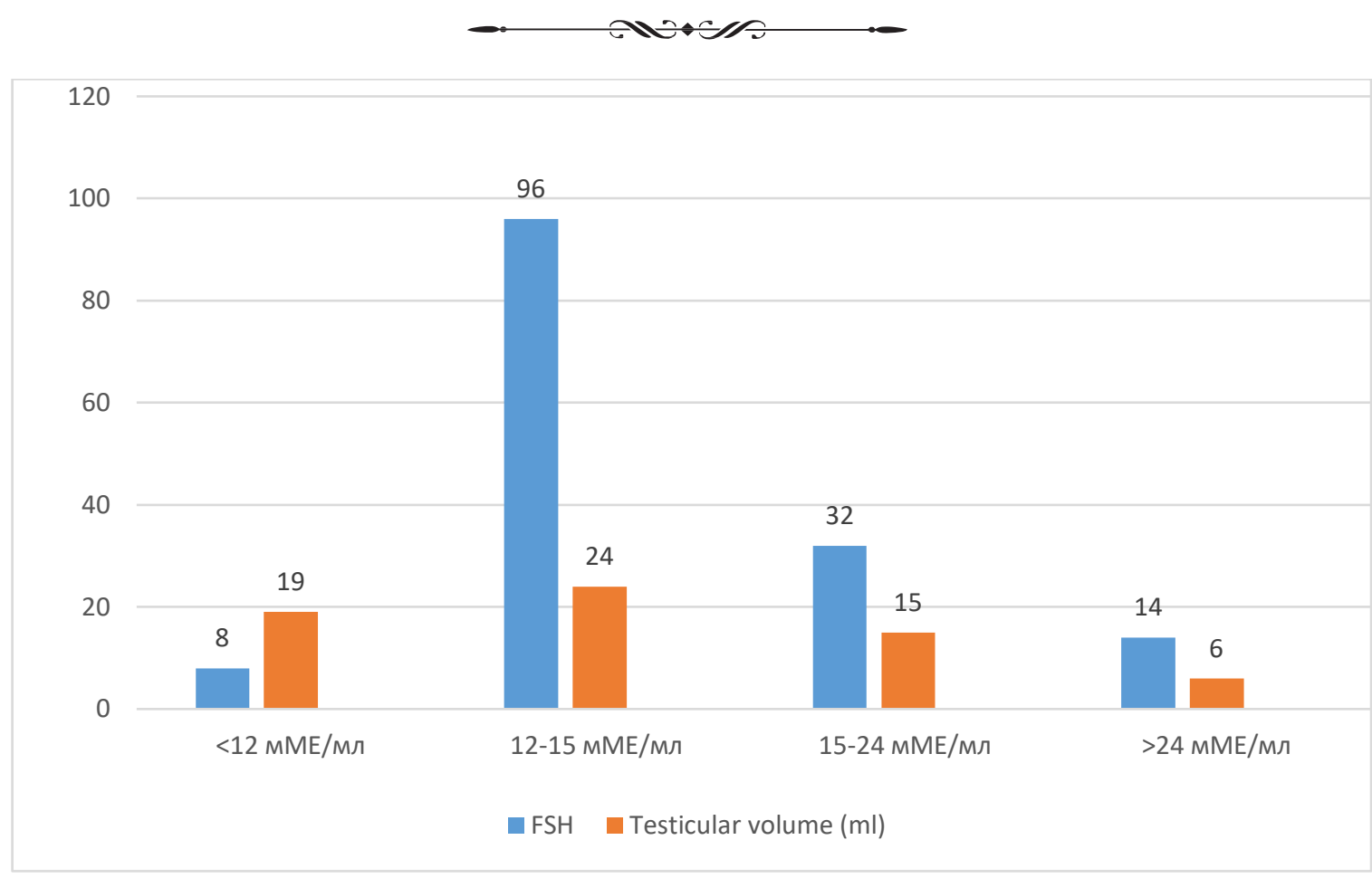

Figure 3. Indicators of FSH level and testicular volume.

Follicle stimulating hormone is a gonadotropin that is produced by the anterior pituitary gland. The role of FSH in puberty is especially important, as this hormone initiates spermatogenesis. Therefore, the level of FSH below the norm indicates the defeat of the hypothalamus and pituitary gland, and in excess of the normative values of the content of FSH, the primary lesion of the genital glands is diagnosed. FSH stimulates proliferation of Sertoli cells of prepubertal and determines their finite number, which then determines the size of the testes and the quality of spermatogenesis.

Conclusions: endocrine disorders occur in 36\% of men with infertility. Indicators of FSH can serve as a guide to the preservation of spermatogenesis.

\section{REFERENCES}

1. Gromov A.I., Bujlov V.M. Radiation diagnostics and therapy in urology: national manual / M.: GOEHTARMedia, 2011. 544p.

2. Kulchenko N.G. Oxidative stress in the development of non-obstructive azoospermia / / Difficult patient. 2017;15(4-5):44-46.

3. Kulchenko N.G. Distruption of spermatogenesis. Morphological aspects. RUDN Journal of Medicine. 2018;22 (3), 265-271.

4. Myandina G.I., Alhedjoj H., Tarasenko E.V., Kulchenko N.G. The influence of the polymorphism G-105A SEPS1 gene on the reproductive function of men // Technology of living systems. 2017;14(6):31-34 .

5. Gnessi L., Scarselli F., Minasi M.G., Mariani S., Lubrano C., Basciani S., Greco P.F., Watanabe M., Franco G. Testicular histopathology, semen analysis and FSH, predictive value of sperm retrieval: supportive counseling in case of reoperation after testicular sperm extraction (TESE). BMC Urology. 2018;18:63-68.

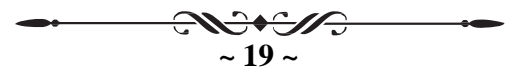

